\title{
Development and Characterization of Microsatellite Markers for Common
} Buckwheat

\author{
Takehiko Konishi*1), Hiroyoshi Iwata' ${ }^{2}$, Kazutoshi Yashiro ${ }^{3)}$, Yoshihiko Tsumura ${ }^{4)}$, Ryo Ohsawa), \\ Yasuo Yasui ${ }^{1)}$ and Ohmi Ohnishi' \\ 1) Laboratory of Crop Evolution, Graduate School of Agriculture, Kyoto University, Nakajo 1, Mozume, Muko, Kyoto 617-0001, Japan \\ 2) Department of Information Science and Technology, National Agricultural Research Center, 3-1-1 Kannondai, Tsukaba, Ibaraki 305- \\ 8666, Japan \\ 3) Plant Biotechnology Institute, Ibaraki Agriculture Center, 3165-1 Ago, Iwama, Nishi-Ibaraki, Ibaraki 319-0292, Japan \\ 4) Department of Forest Genetics, Forestry and Forest Products Research Institute, 1 Matsunosato, Tsukuba, Ibaraki 305-8687, Japan \\ 5) Graduate School of Life and Environmental Sciences, University of Tsukuba, 1-1-1 Tennoudai, Tsukuba, Ibaraki 305-8572, Japan
}

\begin{abstract}
So far, only five microsatellite markers have been developed in common buckwheat (Fagopyrum esculentum). The purpose of the present study was to develop a larger number of microsatellite markers in common buckwheat. By sequencing 2785 clones from the libraries, which were enriched for $(C T)_{n}$ and $(G T)_{n}$ repeats using magnetic particles, it was shown that 1483 clones contained microsatellites, of which 352 had unique sequences. Primer pairs were designed for 237 of the microsatellite loci, of which 180 primer pairs each amplified PCR products. Fifty-four primer pairs that each amplified a clear PCR product of the expected size were evaluated for their ability to detect variations in common buckwheat populations and to be utilized in seven related Fagopyrum species. Forty-eight (88.9\%) out of the 54 microsatellite markers tested were found to be highly variable (the average number of alleles was 12.2 and the average polymorphism information content (PIC) was 0.79 ) in a population of cultivated buckwheat. This PIC value was comparatively large when compared with the average values for microsatellite markers reported for other crops. A high rate of successful amplification of common buckwheat microsatellite markers was observed in closely related species; in particular all the 54 loci were successfully amplified in the wild ancestor of cultivated common buckwheat. The microsatellite markers developed in the present study should contribute to the promotion of molecular breeding in common buckwheat.
\end{abstract}

Key Words: Fagopyrum esculentum, cross-specific amplification, cymosum group, simple sequence repeats (SSRs).

\section{Introduction}

The genus Fagopyrum consists of two major phylogenetic groups, the cymosum group and the urophyllum group. The cymosum group, characterized by large seeds, contains all the useful Fagopyrum species, including the two cultivated species, common buckwheat ( $F$. esculentum Moench) and Tartary buckwheat ( $F$. tataricum Gaert.) (Ohnishi and Matsuoka 1996, Yasui and Ohnishi 1998). As for the other members of the cymosum group, F. homotropicum Ohnishi, a self-compatible wild species, has been successfully crossed with common buckwheat (Campbell 1995, Wang et al. 2002), and is now considered to be an important genetic resource for the improvement of cultivated common buckwheat. Fagopyrum cymosum Meisn., another wild species, has been utilized as a medicinal plant in China and Southeast

Communicated by M. Yano

Received November 16, 2005. Accepted March 7, 2006.

*Corresponding author (e-mail: takehikokonishi@yahoo.co.jp)
Asia (Anderson 1986).

The origin of cultivated common buckwheat has been studied by the analysis of the diffusion routes of cultivated common buckwheat using RAPD markers (Murai and Ohnishi 1996), by the determination of the allozyme diversity of the cultivated populations (Ohnishi 1998) and by the analysis of the genetic relationships between cultivated populations and natural populations of wild common buckwheat using AFLP markers (Konishi et al. 2005). During the course of these studies, several issues that were difficult to solve by the use of allozymes as well as of RAPD and AFLP markers, were identified, for example, the determination of the center of genetic diversity, detailed genetic relationships among cultivated populations, and gene flow between cultivated populations and natural populations of wild common buckwheat. If more powerful (more polymorphic) markers became available, it would be possible to address these issues, so that studies on the origin of cultivated common buckwheat could progress more rapidly.

A microsatellite, also called a simple sequence repeat 
(SSR) which is a sequence consisting of tandem repeats of a short nucleotide motif, is abundantly distributed throughout eukaryotic genomes (Litt and Luty 1989, Weber and May 1989). Microsatellite markers are a powerful tool for the analysis of wide genetic variations within or among populations because of their co-dominance, multiallelism and high variability (Litt and Luty 1989, Tautz 1989). In many crops, they have been developed and used for the study of genetic diversity and population structure, for example in durum wheat (Thuillet et al. 2005), maize (Vigouroux et al. 2005) and rice (Garris et al. 2005).

Iwata et al. (2001) who developed microsatellite markers for common buckwheat, observed a significant genetic differentiation among Japanese common buckwheat cultivars (Iwata et al. 2005). However, only five microsatellite markers were developed by Iwata et al. (2001). In the present study, we developed a large number of microsatellite markers for common buckwheat and evaluated their variation within a common buckwheat population. Since the development of microsatellite markers is expensive and timeconsuming, the utility of the markers would be increased if the microsatellite markers developed for common buckwheat could be used for related species. Therefore, we also determined whether the microsatellite markers developed for common buckwheat could be used for other Fagopyrum species of the cymosum group.

\section{Materials and Methods}

\section{Plant materials and DNA extraction}

An individual of $F$. esculentum ssp. esculentum from the Boke population (accession number, C9701) in Sichuan province, China was the source of the DNA used to construct a microsatellite-enriched library. An artificial population of common buckwheat consisting of 34 individuals, one individual each from 34 worldwide accessions (19 Chinese landraces and 15 landraces from 15 countries) was used to analyze the microsatellite variation.

To determine whether the microsatellite markers which were developed for cultivated common buckwheat could be utilized in related Fagopyrum species, one individual was used for each of the following seven taxa; F. esculentum ssp. ancestrale (C2009), diploid and tetraploid $F$. homotropicum (C9740, C9737), F. tataricum ssp. tataricum (C9722), F. tataricum ssp. potanini (C9022), diploid and tetraploid F. cymosum (C8924, B9103). F. esculentum ssp. ancestrale and $F$. tataricum ssp. potanini are the wild ancestors of cultivated common and Tartary buckwheat, respectively (Ohnishi 1998).

The total DNA of each individual plant was extracted from fresh leaf tissue using Plant DNAzol ${ }^{\circledR}$ Reagent (Invitrogen Corp., Carlsbad, CA, USA).

\section{Isolation of microsatellites and primer design}

A library enriched for $(\mathrm{GT})_{\mathrm{n}}$ dinucleotide was constructed according to the method of Tani et al. (2004) using magnetic particles. The hybridizing probe used for enrichment consisted of the 5'-biotinylated $(\mathrm{GT})_{15}$ oligonucleotide. Cloned DNAs from the library were sequenced using an ABI PRISM 3100 Genetic Analyzer with a BigDye Terminator ver. 3 (PE Applied Biosystems, Foster City, CA, USA). In addition, many cloned DNAs from the $(\mathrm{CT})_{\mathrm{n}^{-}}$ enriched library constructed by Iwata et al. (2001), of which sequences had not been elucidated, were also sequenced, and used in this study. Microsatellites, according to Weber (1990), were identified by checking nucleotide sequence data. Duplicated sequences among clones were detected by homology search of the clones in the libraries. If homologous sequences were found, only one unique sequence was kept for primer design. For each unique sequence, primer pairs were designed using the computer software OLIGO (National Biosciences. Inc., Plymouth, MN, USA) and Primer3 listed at http://waldo.wi.mit.edu/egi-bin/primer/ primer3 (Whitehead Institute for Biomedical Research).

\section{PCR amplification and detection of PCR products}

After optimization of the PCR conditions, PCR amplification was performed in a $15 \mu \mathrm{l}$ reaction solution containing $0.2 \mu \mathrm{M}$ of each primer, $200 \mu \mathrm{M}$ of each dNTP, $10 \mathrm{mM}$ of Tris- $\mathrm{HCl}\left(\mathrm{pH} \mathrm{8.3),} 50 \mathrm{mM}\right.$ of $\mathrm{KCl}, 1.5 \mathrm{mM}$ of $\mathrm{MgCl}_{2}, 0.5 \mathrm{U}$ of Taq DNA polymerase (New England Biolabs, Beverly, MA, USA), and $15 \mathrm{ng}$ of template DNA using an I-Cycler (BIO-RAD, Hercules, CA, USA) under the following conditions: $2 \mathrm{~min}$ at $94^{\circ} \mathrm{C}, 30$ cycles of $30 \mathrm{sec}$ at $94^{\circ} \mathrm{C}, 30 \mathrm{sec}$ at $46-69^{\circ} \mathrm{C}$ and $60 \mathrm{sec}$ at $72^{\circ} \mathrm{C}$, followed by $3 \mathrm{~min}$ at $72^{\circ} \mathrm{C}$. The annealing temperature used for each primer pair is listed in Appendix 1. Microsatellite alleles were identified by running the PCR products on a denaturing $5 \%(\mathrm{w} / \mathrm{v})$ ureapolyacrylamide (19:1 acrylamide:Bis, $8 \mathrm{M}$ urea) gel in a TBE buffer at $100 \mathrm{~W}$ for 2 hours and visualized by a silverstaining method using SILVER SEQUENCE TM DNA Staining Reagents (Promega Corp., Madison, WI, USA).

\section{Analysis of microsatellite variation}

Fifty-four primer pairs that yielded an amplification product of the expected size were analyzed for their variability in the artificial population comprizing 34 individuals. For the primer pairs that detected polymorphism, the number of alleles (NA) and the polymorphism information content (PIC) were calculated as follows: $\mathrm{PIC}=1-\Sigma_{\mathrm{i}} p_{i}{ }^{2}$, where $p_{i}$ is the frequency of the $i$-th allele in the sample examined (Anderson et al. 1993).

\section{Amplification in related Fagopyrum species}

The fifty-four primer pairs which were used for the analysis of microsatellite variability were also analyzed for their use in the seven Fagopyrum species or subspecies which are closely related to common buckwheat. PCR amplification was performed using the same protocol as that described above, and repeated at least twice when no amplification was observed on the first attempt. Since the required amount of DNA was not obtained to test all the primer pairs 
for diploid and tetraploid $F$. cymosum, only 30 and nine primer pairs were evaluated for diploid and tetraploid F. cymosum, respectively.

\section{Results and Discussion}

DNA sequences were determined for 1875 and 910 clones from the two libraries, enriched for $(\mathrm{CT})_{\mathrm{n}}$ and $(\mathrm{GT})_{\mathrm{n}}$ dinucleotides, respectively. Of the 1875 clones from the library enriched for $(\mathrm{CT})_{\mathrm{n}}, 1079(57.5 \%)$ were found to contain microsatellites. Two hundred and forty-five clones each containing a unique sequence were identified. Although the remaining 834 clones were duplicated more than once in the enriched library, they could be classified into 148 groups based on unique sequences. In total, 393 unique sequences were thus obtained. For the library enriched by $(\mathrm{GT})_{\mathrm{n}}, 404$ (44.4\%) of the 910 clones were found to contain microsatellites. One hundred and six clones contained unique sequences, and the remaining 298 clones were classified into 59 groups based on unique sequences. Therefore, in total, 165 unique sequences were obtained.

Although 393 and 165 unique sequences were obtained from the libraries enriched for $(\mathrm{CT})_{\mathrm{n}}$ and $(\mathrm{GT})_{\mathrm{n}}$, respectively, many of the sequences were duplicated between the two libraries. By removing all the unnecessary duplicated sequences, 352 unique sequences were thus obtained, of which 237 sequences could be used to design primers. Of the 237 primer pairs, 180 pairs amplified PCR products (see Appendix 1), while the remaining 57 pairs did not amplify any PCR products even at the lower annealing temperature of $46^{\circ} \mathrm{C}$. Of the 180 primer pairs which amplified PCR products, 54 pairs each amplified a clear PCR product of the expected size (Fig. 1a). We evaluated these 54 markers for their ability to detect variation in a common buckwheat pop-

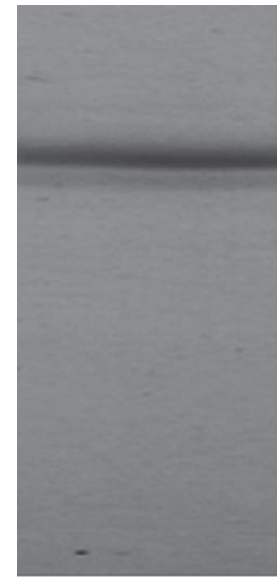

(a)

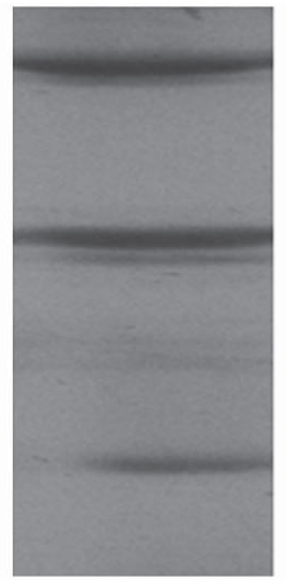

(b)

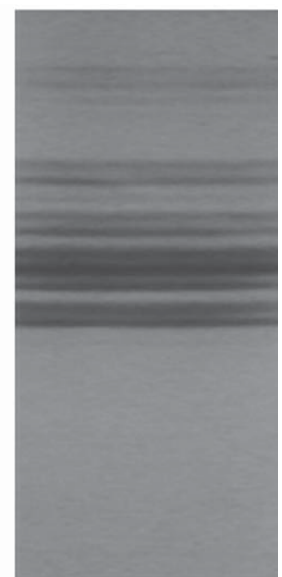

(c)
Fig. 1. PCR products separated on $5 \%$ denaturing polyacrylamide gels and visualised by silver staining. (a) PCR products with a single clear band of the expected size, (b) PCR products with more than one band and (c) PCR products with ladder-like bands. ulation, and for their ability to be utilized in the 7 related Fagopyrum species. The remaining 126 pairs amplified more than one fragment (Fig. 1b,c). These pairs may amplify several sites other than the target site. The use of these 126 pairs is not convenient for detecting genetic variation because the amplified loci may not be homologous among individuals.

Among the 54 loci at which a clear PCR product with the expected size was amplified, 48 (88.9\%) loci exhibited microsatellite variation in the experimental common buckwheat population. Figure 2 shows some examples of the loci displaying variation (Fes1585 and Fes3331). The number of alleles per polymorphic locus, NA, ranged from two to 30 with an average value of 12.2 , while the PIC ranged from 0.42 to 0.95 with an average value of 0.79 (Table 1). Many authors have reported that microsatellite loci with a larger number of repeats of motif showed a wider variation than microsatellite loci with a smaller number of repeats of motif for such crops as barley (Ramsay et al. 2000), maize (Sharopova et al. 2002) and tomato (He et al. 2003). In common buckwheat, a significant correlation was detected between the number of $(\mathrm{GA} / \mathrm{CT})$ repeats in the microsatellites and NA and PIC (correlation coefficient $(r)=0.654(\mathrm{P}<$ $0.001)$ and $r=0.584(\mathrm{P}<0.001)$, respectively). Microsatellites with the other types of repeats were not investigated because the number of samples was small. The average PIC value (0.79) was comparatively large when compared with that for other crops; namely 0.37 for tomato (He et al. 2003), 0.59 for rice (Temnykh et al. 2000), 0.62 for maize (Smith et al. 1997) and 0.71 for wheat (Prasad et al. 2000). This may be due to the fact that the artificial population consisted of 34 individuals from a world-wide collection of landraces, i.e. the population was expected to contain variation at the species level.

Of the 54 microsatellite markers which were evaluated for their ability to be utilized in the seven related Fagopyrum species, two loci (Fes1693 and Fes2459) could be amplified in all the seven taxa (Table 1). Many authors have reported that the transferability of the microsatellite markers decreased with the increase in the genetic distance between taxa in rapeseed (Lagercrantz et al. 1993) and soybean (Peakall et al. 1998). In the Fagopyrum species, this can be observed by comparing the number of different nucleotides in $r b c \mathrm{~L}$ gene sequences (Yasui and Ohnishi 1998) and the percentage of successful amplification (Table 2). The percentage of successful amplification in $F$. esculentum ssp. ancestrale, which showed exactly the same $r b c \mathrm{~L}$ sequence as common buckwheat, was $100 \%$. In a more distantly related species, diploid and tetraploid forms of $F$. cymosum were different from $F$. esculentum ssp. esculentum at seven nucleotides. The percentage of successful amplification was $36.7 \%$ and $33.3 \%$ in diploid and tetraploid forms of F. cymosum, respectively. The rate of transferability in tetraploids was slightly lower than that in diploid counterparts, namely $79.6 \%$ vs. $68.5 \%$ in F. homotropicum and $36.7 \%$ vs. $33.3 \%$ in F. cymosum (Table 2), which was consistent with previous reports indicating that the percentage of primer 
Table 1. Polymorphism parameters (NA and PIC) and transferability of 54 microsatellite markers

\begin{tabular}{|c|c|c|c|c|c|c|c|c|c|}
\hline Locus & $\mathrm{NA}^{1)}$ & PIC & $\mathrm{ANC}^{2)}$ & HOM $2 x$ & HOM4x & TAT & POT & CYM2x & CYM4x \\
\hline Fes1094 & 20 & 0.92 & $\bigcirc^{3)}$ & 0 & 0 & $\bigcirc$ & $x$ & - & - \\
\hline Fes 1284 & 1 & - & $\bigcirc$ & $\bigcirc$ & $\bigcirc$ & $\bigcirc$ & $x$ & $\bigcirc$ & - \\
\hline Fes 1286 & 22 & 0.93 & $\bigcirc$ & $\bigcirc$ & $x$ & $\bigcirc$ & $\bigcirc$ & $x$ & $x$ \\
\hline Fes 1303 & 28 & 0.95 & $\bigcirc$ & $\bigcirc$ & $\bigcirc$ & 0 & $x$ & $x$ & - \\
\hline Fes 1322 & 24 & 0.95 & $\bigcirc$ & $x$ & $x$ & $x$ & $x$ & - & - \\
\hline Fes 1346 & 9 & 0.84 & $\bigcirc$ & $\times$ & $\times$ & 0 & $x$ & $\times$ & - \\
\hline Fes 1362 & 10 & 0.82 & $\bigcirc$ & $x$ & 0 & $\times$ & $x$ & $x$ & $x$ \\
\hline Fes 1368 & 15 & 0.89 & $\bigcirc$ & $\bigcirc$ & $\bigcirc$ & $\bigcirc$ & $x$ & - & - \\
\hline Fes1394 & 11 & 0.75 & $\bigcirc$ & $\bigcirc$ & $\bigcirc$ & 0 & $x$ & - & - \\
\hline Fes1407 & 23 & 0.92 & 0 & 0 & 0 & $x$ & $x$ & - & - \\
\hline Fes1409 & 8 & 0.73 & 0 & $x$ & $\bigcirc$ & $x$ & $x$ & - & - \\
\hline Fes1475 & 15 & 0.83 & $\bigcirc$ & $\times$ & $x$ & $\bigcirc$ & $x$ & $x$ & - \\
\hline Fes1497 & 18 & 0.92 & $\bigcirc$ & $x$ & $x$ & 0 & $x$ & - & - \\
\hline Fes 1498 & 6 & 0.64 & $\bigcirc$ & $\bigcirc$ & 0 & $x$ & $x$ & - & - \\
\hline Fes 1501 & 5 & 0.65 & $\bigcirc$ & $\bigcirc$ & 0 & 0 & 0 & - & - \\
\hline Fes 1582 & 14 & 0.89 & $\bigcirc$ & $x$ & $x$ & $x$ & $x$ & - & - \\
\hline Fes 1583 & 5 & 0.62 & 0 & $\bigcirc$ & $\bigcirc$ & 0 & $x$ & $\times$ & - \\
\hline Fes1585 & 4 & 0.63 & $\bigcirc$ & $\bigcirc$ & $\bigcirc$ & 0 & $x$ & - & - \\
\hline Fes 1623 & 13 & 0.83 & $\bigcirc$ & 0 & $\times$ & 0 & $\times$ & - & - \\
\hline Fes 1693 & 1 & - & $\bigcirc$ & 0 & 0 & 0 & 0 & 0 & 0 \\
\hline Fes 1816 & 13 & 0.87 & 0 & 0 & $\bigcirc$ & 0 & $x$ & - & - \\
\hline Fes 1822 & 20 & 0.85 & $\bigcirc$ & $x$ & $x$ & 0 & $\bigcirc$ & 0 & $\bigcirc$ \\
\hline Fes 1840 & 8 & 0.80 & 0 & 0 & 0 & $x$ & $x$ & $x$ & - \\
\hline Fes 1896 & 1 & - & $\bigcirc$ & $x$ & $x$ & $x$ & $x$ & - & - \\
\hline Fes 2328 & 11 & 0.86 & 0 & 0 & 0 & $x$ & $x$ & $x$ & - \\
\hline Fes2459 & 1 & - & 0 & 0 & 0 & 0 & 0 & 0 & 0 \\
\hline Fes2468 & 11 & 0.80 & 0 & $\bigcirc$ & $\bigcirc$ & 0 & 0 & - & - \\
\hline Fes2480 & 13 & 0.78 & 0 & 0 & 0 & $x$ & $x$ & $x$ & - \\
\hline Fes 2507 & 7 & 0.62 & 0 & 0 & $x$ & 0 & $x$ & $x$ & $x$ \\
\hline Fes2644 & 8 & 0.80 & 0 & 0 & $x$ & 0 & $x$ & $x$ & - \\
\hline Fes2741 & 2 & 0.48 & $\bigcirc$ & 0 & $x$ & 0 & $x$ & $x$ & - \\
\hline Fes2743 & 4 & 0.62 & $\bigcirc$ & 0 & 0 & 0 & $x$ & $\times$ & - \\
\hline Fes2751 & 24 & 0.93 & $\bigcirc$ & $\times$ & $\times$ & $\times$ & $x$ & $x$ & - \\
\hline Fes2798 & 9 & 0.72 & $\bigcirc$ & 0 & 0 & $x$ & $x$ & - & - \\
\hline Fes 2802 & 4 & 0.69 & $\bigcirc$ & $\bigcirc$ & $\bigcirc$ & $x$ & $x$ & $x$ & - \\
\hline Fes2809 & 8 & 0.52 & $\bigcirc$ & 0 & $\bigcirc$ & 0 & $\bigcirc$ & $x$ & $x$ \\
\hline Fes2857 & 14 & 0.84 & 0 & 0 & 0 & 0 & $x$ & - & - \\
\hline Fes2900 & 11 & 0.76 & 0 & 0 & 0 & 0 & 0 & 0 & - \\
\hline Fes2976 & 7 & 0.67 & $\bigcirc$ & 0 & $x$ & 0 & $x$ & - & - \\
\hline Fes3075 & 1 & - & 0 & 0 & 0 & 0 & 0 & 0 & - \\
\hline Fes 3108 & 12 & 0.76 & 0 & 0 & $x$ & 0 & $x$ & - & - \\
\hline Fes3141 & 9 & 0.81 & 0 & $\bigcirc$ & $x$ & $x$ & $x$ & - & - \\
\hline Fes3164 & 18 & 0.88 & 0 & 0 & 0 & 0 & $x$ & - & - \\
\hline Fes 3177 & 7 & 0.80 & 0 & 0 & 0 & $x$ & $x$ & - & - \\
\hline Fes 3214 & 15 & 0.86 & 0 & 0 & 0 & 0 & 0 & 0 & - \\
\hline Fes3265 & 14 & 0.90 & $\bigcirc$ & $\bigcirc$ & 0 & 0 & 0 & 0 & $x$ \\
\hline Fes3331 & 7 & 0.82 & $\bigcirc$ & 0 & 0 & 0 & $\bigcirc$ & - & - \\
\hline Fes3508 & 19 & 0.93 & $\bigcirc$ & $\bigcirc$ & $\bigcirc$ & $x$ & $\bigcirc$ & - & - \\
\hline Fes3663 & 8 & 0.75 & $\bigcirc$ & $\bigcirc$ & $\bigcirc$ & $\times$ & $x$ & $\bigcirc$ & - \\
\hline Fes3664 & 10 & 0.80 & 0 & 0 & 0 & 0 & 0 & $\bigcirc$ & $x$ \\
\hline Fes3768 & 7 & 0.78 & $\bigcirc$ & 0 & $\bigcirc$ & $x$ & $\times$ & $x$ & - \\
\hline Fes4036 & 30 & 0.94 & 0 & 0 & 0 & 0 & $x$ & $x$ & - \\
\hline Fes4045 & 5 & 0.42 & 0 & 0 & 0 & $x$ & 0 & 0 & - \\
\hline Fes 4620 & 1 & - & $\bigcirc$ & $\times$ & $x$ & $x$ & $x$ & $x$ & - \\
\hline
\end{tabular}

1) NA: number of alleles, PIC: polymorphic information content

2) ANC: F. esculentum ssp. ancestrale, HOM2x: F. homotropicum (2x), HOM4x: F. homotropicum (4x), TAT: F. tataricum ssp. tataricum, POT: F. tataricum ssp. potanini, CYM2x: F. cymosum (2x) and CYM4x: F. cymosum (4x)

3) $\bigcirc$ : amplified, $X$ : not amplified and $-:$ not tested 
(a) Fes 1585

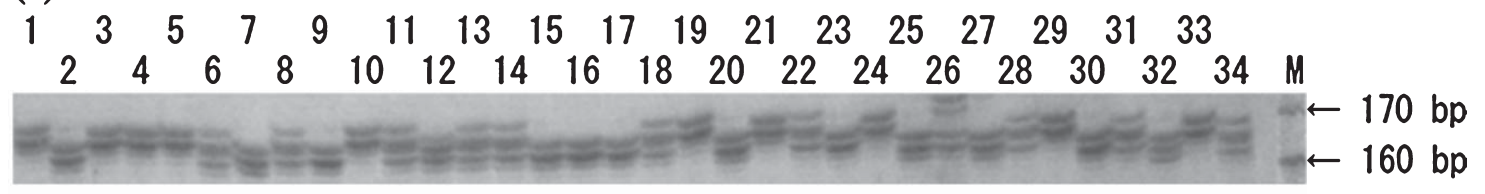

(b) Fes3331

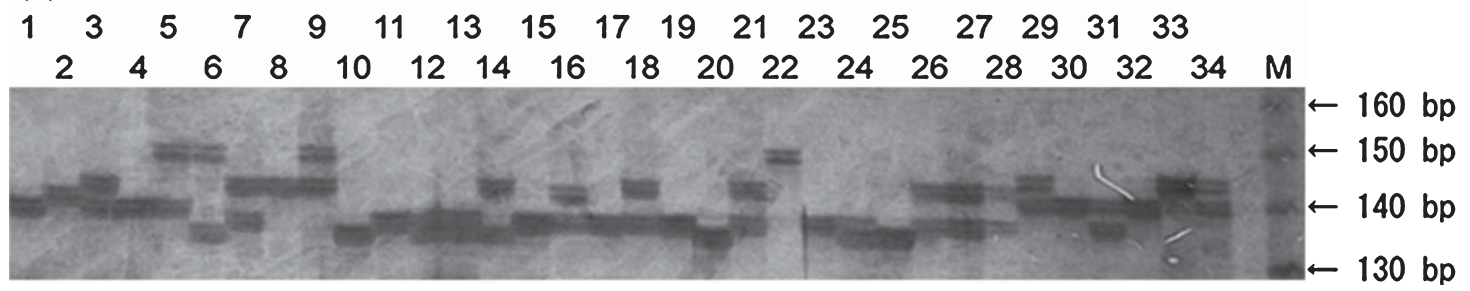

Fig. 2. Polymorphism detected among 34 common buckwheat accessions at a microsatellite locus, (a) Fes1585 and (b) Fes3331. M stands for the size marker lanes. Lanes 1 to 34, as numbered from left to right, correspond to the 34 individuals examined.

Table 2. Percentage of successful amplification and number of different nucleotides in $r b c \mathrm{~L}$ gene sequence between F. esculentum ssp. esculentum and the related Fagopyrum species or subspecies

\begin{tabular}{|c|c|c|}
\hline & Percentage of successful amplification & Number of different nucleotides ${ }^{1)}$ \\
\hline$F$. esculentum ssp. ancestrale & $100.0 \%$ & 0 \\
\hline Diploid F. homotropicum & $79.6 \%$ & 1 \\
\hline Tetraploid $F$. homotropicum & $68.5 \%$ & - \\
\hline F. tataricum ssp. tataricum & $63.0 \%$ & 1 \\
\hline F. tataricum ssp. potanini & $25.9 \%$ & 7 \\
\hline Diploid $F$. cymosum & $36.7 \%$ & 7 \\
\hline Tetraploid $F$. cymosum & $33.3 \%$ & 7 \\
\hline \multicolumn{3}{|c|}{$\begin{array}{l}\text { 1) Sequence data of } r b c \mathrm{~L} \text { gene for six species or subspecies were obtained from DDBJ database. Accession numbers } \\
\text { in DDBJ database: } F \text {. esculentum ssp. ancestrale, AB000310; diploid F. homotropicum, AB000312; F. tataricum } \\
\text { ssp. tataricum, AB000319; F. tataricum ssp. potanini, AB000318; diploid F. cymosum, AB000307; tetraploid } \\
\text { F. cymosum, AB000304. (-): Sequence data for tetraploid } F \text {. homotropicum could not be obtained. A total of } \\
1,428 \text { nucleotides were compared. }\end{array}$} \\
\hline
\end{tabular}

pairs that successfully amplified microsatellite loci was lower in the polyploid species than in their diploid counterparts in tetraploid alfalfa (Diwan et al. 1997) and hexaploid wheat (Roder et al. 1995). The low efficiency of PCR amplification in the tetraploids may be due to the complexity of the polyploid genome (Roder et al. 1995).

In conclusion, it was clearly shown that the 54 microsatellite markers displayed a high variability in common buckwheat and high transferability for use in closely related Fagopyrum species. We have recently detected and analyzed microsatellite variations in common buckwheat and its wild ancestor populations, and showed that gene flow between cultivated and wild populations was not important for determining the phylogenetic relationships between them (Konishi and Ohnishi, unpublished data). Furthermore, we have recently constructed a linkage map based on the 180 microsatellite markers developed in the present study (54 markers listed above together with the 126 markers that amplified more than one fragment). This may contribute to the promotion of molecular breeding in common buckwheat. The results related to the construction of a linkage map of microsatellite markers will be published elsewhere.

\section{Acknowledgements}

We thank Dr. K. Yamane, Osaka Prefecture University, for providing the DNA from diploid and tetraploid F. cymosum, and Dr. Clayton Campbell, Kade Research Ltd., Morden, Manitoba, Canada, for reading the manuscript and correcting the English. Contribution No. 126 from Laboratory of Crop Evolution, Graduate School of Agriculture, Kyoto University

\section{Literature Cited}

Anderson,E.F. (1986) Ethnobotany of hilltribes of northern Thailand. I. Medicinal plants of Akha. Economic Bot. 40: 38-53.

Anderson,J.A., G.A.Churchill, J.E. Autrique, S.D.Tanksley and M.E. Sorrells (1993) Optimizing parental selection for genetic linkage maps. Genome 36: 181-186.

Campbell (1995) Inter-specific hybridization in the genus Fagopyrum. Proc. $6^{\text {th }}$ Intl. Symp. Buckwheat at Ina, Japan, 255-263.

Diwan, N., A.A. Bhagwat, G.B.Bauchan and P.B.Cregan (1997) 
Simple sequence repeat DNA markers in alfalfa and perennial and annual Medicago species. Genome 40: 887-895.

Garris,A.J., T.H.Tai, J.Coburn, S.Kresovich and S.McCouch (2005) Genetic structure and diversity in Oryza sativa L.. Genetics 169: 1631-1638.

He,C., V.Poysa and K.Yu (2003) Development and characterization of simple sequence repeat (SSR) markers and their use in determining relationships among Lycopersicon esculentum cultivars. Theor. Appl. Genet. 106: 363-373.

Iwata,H., K.Imon, K.Yoshimura, Y.Tsumura and R.Ohsawa (2001) Genetic diversity of common buckwheat varieties in Japan based on microsatellite markers. Proc. $8^{\text {th }}$ Intl. Symp. Buckwheat at Chunchon, Korea, 240-247.

Iwata,H., K.Imon, Y.Tsumura and R.Ohsawa (2005) Genetic diversity among Japanese indigenous common buckwheat (Fagopyrum esculentum) cultivars as determined from amplified fragment length polymorphism and simple sequence repeat markers and quantitative agronomic traits. Genome 48: 367-377.

Konishi,T., Y.Yasui and O.Ohnishi (2005) Original birthplace of cultivated common buckwheat inferred from genetic relationships among cultivated populations and natural populations of wild common buckwheat revealed by AFLP analysis. Genes Genet. Syst. 80: 113-119.

Lagercrantz,U., H.Ellegren and L.Andersson (1993) The abundance of various polymorphic microsatellite motifs differs between plants and vertebrates. Nucleic Acids Res. 21: 1111-1115.

Litt,M. and J.A.Luty (1989) A hypervariable microsatellite revealed by in vitro amplification of a dinucleotide repeat within the cardiac muscle actin gene. Am. J. Human Genet. 44: 397-401.

Murai,M. and O.Ohnishi (1996) Population genetics of cultivated common buckwheat, Fagopyrum esculentum Moench. X. Diffusion routes revealed by RAPD markers. Genes Genet. Syst. 71: 211-218.

Ohnishi,O. (1998) Search for the wild ancestor of buckwheat III. The wild ancestor of cultivated common buckwheat, and of Tartary buckwheat. Economic Bot. 52: 123-133.

Ohnishi,O. and Y.Matsuoka (1996) Search for the wild ancestor of buckwheat II. Taxonomy of Fagopyrum (Polygoneae) species based on morphology, isozymes and cpDNA variability. Genes Genet. Syst. 71: 383-390.

Peakall,R., S.Gilmore, W.Keys, M.Morgante and A.Rafalski (1998) Cross-species amplification of soybean (Glycine max) simple sequence repeats (SSRs) within the genus and other legume genera: implications for the transferability of SSRs in plants. Mol. Biol. Evol. 15: 1275-1287.

Prasad, M., R.K. Varshney, J.K. Roy, H.S. Balyan and P.K. Gupta (2000) The use of microsatellites for detecting DNA polymorphism, genotype identification and genetic diversity in wheat. Theor. Appl. Genet. 100: 584-592.
Ramsay,L., M.Macaulay, K.degli Ivanissevich, S.Maclean, L.Cardle et al. (2000) A simple sequence repeat-based linkage map of barley. Genetics 156: 1997-2005.

Roder,M.S., J.Plaschke, S.U.Konig, A.Bormer, M.E. Sorells, S.D. Tanksley and M.W.Ganal (1995) Abundance, variability and chromosomal location of microsatellites in wheat. Mol. Gen. Genet. 246: 327-333.

Sharopova, N., M.D.Mcmullen, L.Schultz, S.Schroeder, H.SanchezVilleda et al. (2002) Development and mapping of SSR markers for maize. Plant Mol. Biol. 48: 463-481.

Smith,J.S.C., E.C.L.Chin, H.Shu, O.S.Smith, S.J.Wall, M.L.Senior, S.E.Mitchell, S.Kresovich and J.Ziegle (1997) An evaluation of the utility of SSR loci as molecular markers in maize (Zea mays L.); comparisons with data from RFLPs and pedigree. Theor. Appl. Genet. 95: 163-173.

Tani, N., T.Takahashi, T.Ujino-Ihara, H.Iwata, K.Yoshimura and Y.Tsumura (2004) Development and characteristics of microsatellite markers for sugi (Cryptomeria japonica D. Don) derived from microsatellite-enriched libraries. Am. For. Sci. 61: $569-575$.

Tautz,D. (1989) Hypervariability of simple sequences as general source for polymorphic DNA markers. Nucleic Acids Res. 17: 6463-6471.

Temnykh,S., W.D.Park, N.Ayres, S.Cartinhour, N.Hauck, L.Lipovich, Y.G.Cho, T.Ishii and S.R.McCouch (2000) Mapping and genome organization of microsatellite sequences in rice (Oryza sativa L.). Theor. Appl. Genet. 100: 697-712.

Thuillet,A.-C., T.Bataillon, S.Poirier, S.Santoni and J.L.David (2005) Estimation of long-term effective population sizes through the history of durum wheat using microsatellite data. Genetics 169 : 1589-1599.

Vigouroux,Y., S.Mitchell, Y.Matsuoka, M.Hamblin, S.Kresovich, J.Stephen, C.Smith, J.Jaqueth, O.S.Smith and J.Doebley (2005) An analysis of genetic diversity across the maize genome using microsatellites. Genetics 169: 1617-1630.

Wang, Y., R.Scarth and C.Campbell (2002) Comparison between diploid and tetraploid forms of Fagopyrum homotropicum in intraspecific and interspecific crossability and cytological characteristics. Fagopyrum 19: 23-29.

Weber,J.L. (1990) Informativeness of human $(\mathrm{dC}-\mathrm{dA})_{\mathrm{n}}$, (dG-dT) polymorphisms. Genomics 7: 524-530.

Weber,J.L. and P.E.May (1989) Abundant class of human DNA polymorphism which can be typed using the polymerase chain reaction. Am. J. Human Genet. 44: 388-396.

Yasui, Y. and O. Ohnishi (1998) Interspecific relationships in Fagopyrum (Polygonaceae) revealed by the nucleotide sequences of the $r b c L$ and $a c c D$ genes and their intergenic region. Am. J. Bot. 85: 1134-1142. 
Appendix 1. Primer sequence, repeat type, expected fragment size, annealing temperature of 180 microsatellite markers

\begin{tabular}{|c|c|c|c|c|c|c|}
\hline Locus & Forward primer $\left(5^{\prime}\right.$ to $\left.3^{\prime}\right)$ & Reverse primer ( $5^{\prime}$ to $\left.3^{\prime}\right)$ & Repeat type & $\begin{array}{l}\text { Fragment } \\
\text { size (bp) }\end{array}$ & $\begin{array}{l}\text { Annealing } \\
\text { temp. }\left({ }^{\circ} \mathrm{C}\right)\end{array}$ & $\begin{array}{l}\text { Evaluation } \\
\text { test* }\end{array}$ \\
\hline Fes 1002 & CAGGGAGGAGAAGGAAAGAGAT & СССТТСТСТСААСТСАССТСТА & $(\mathrm{GA})_{7}$ & 189 & 64 & \\
\hline Fes 1012 & CTTCCTTTCTAGCCGTTACA & GAAGGTTGAGTCGCACAGGT & $(\mathrm{TC})_{8}$ & 100 & 59 & \\
\hline Fes 1051 & GAAGGAGCATAAGAGAGGAT & CGTTTCCCGCCTACA & $(\mathrm{GA})_{22}$ & 200 & 55 & \\
\hline Fes1061 & TGCACTACGGTTTGAGTATT & AGGGTTGGCTGACTTATGTT & $(\mathrm{GA})_{10} \mathrm{GG}(\mathrm{GA})_{11}$ & 201 & 59 & \\
\hline Fes1094 & GAAGCCTTGGAAGAAGTGAAAT & TAAAGCTCATCCCAATATGCAA & $(\mathrm{AG})_{37}$ & 160 & 61 & $\mathrm{O}$ \\
\hline Fes 1264 & GCTGTCTCCAGTCCGCATCC & GCTCTTGACCCTTGAATACC & $(\mathrm{GA})_{9}$ & 186 & 63 & \\
\hline Fes 1284 & GAATACACCATTCGGAACAAGA & ATCATCCTCCTTTGAGTCACCT & $(\mathrm{ATG})_{4}$ & 114 & 61 & $\bigcirc$ \\
\hline Fes 1286 & CCATATTCTACTTTCCGACC & GAAGCACAAGGAAATGAGGG & $(\mathrm{GA})_{25}$ & 180 & 62 & 0 \\
\hline Fes 1298 & TTGTTGTAGTTGTTGTTTCG & ATTGAAGGAGCTATTGATGG & $(\mathrm{TTG})_{6}$ & 123 & 62 & \\
\hline Fes 1303 & AGGAGACGGGAGAGAAGCAG & GGATGTTTGGGTGATTTCAG & $(\mathrm{GA})_{31}$ & 225 & 60 & $\bigcirc$ \\
\hline Fes 1307 & AGAGGAACAAGAAGCACCAT & CCCTCAGTTGCTTGACCTAT & $(\mathrm{GA})_{7}$ & 155 & 63 & \\
\hline Fes 1308 & ACTCTTGACCCGTGTTTTACCT & TAGCGTGAGAGAGAGAGAGAGA & $(\mathrm{CT})_{20}$ & 92 & 59 & \\
\hline Fes 1309 & GTGTTGAATGTATGCACCAG & TTGACAAGTCATGGACAAGG & $(\mathrm{GA})_{10}$ & 155 & 63 & \\
\hline Fes 1322 & AAGCATTCATTCATTCATTC & GAGTTTGTTGTGTTTGGAGG & $(\mathrm{CT})_{31}$ & 177 & 58 & $\bigcirc$ \\
\hline Fes 1323 & TGAAGGAGGGAAGAAGATGAAT & TCCCTCTTATGGATTGGAGACT & $(\mathrm{AAG})_{4}$ & 94 & 62 & \\
\hline Fes 1326 & GACCCTTCGTTAGTAGACCA & GGAGGAAGAGAAGAGAAAGT & $(\mathrm{TC})_{11}$ & 146 & 61 & \\
\hline Fes 1346 & GGTGCAAACTAACATGAATG & GCTGAGTCTCGGGATTTATT & $(\mathrm{GA})_{17}$ & 202 & 60 & $\bigcirc$ \\
\hline Fes 1347 & ATTCCCCCAAGAACACCCAC & CCCTTGTTGGAGTATAAGAG & $(\mathrm{GA})_{41}$ & 167 & 59 & \\
\hline Fes 1354 & TCCATGGCTTACACAACACT & CTTGACGCTTCCTGACCTCT & $(\mathrm{GA})_{23}$ & 253 & 62 & \\
\hline Fes 1356 & ACGGAAGTGCAAGGAAGGAA & ATGAGAAACAACCCAATCTG & $(\mathrm{GA})_{30}$ & 183 & 61 & \\
\hline Fes 1362 & CCCAGCGTGTGATGATTGAC & CGGCATGCGAAACGAAGGAG & $(\mathrm{CT})_{8}$ & 276 & 63 & $\bigcirc$ \\
\hline Fes 1367 & GTTCATTTCAGCAACTAACA & TCATACCATCACCCAACAAG & $(\mathrm{CT})_{22}$ & 175 & 61 & \\
\hline Fes 1368 & CAACCACTCAAAGCCTCATC & CTTTCATATCCСТАACACAC & $(\mathrm{CT})_{34}$ & 177 & 55 & $\bigcirc$ \\
\hline Fes 1393 & GCGGCTAGTCTGGGAAGAAA & CTCGAATCCTTGGCAATCAC & $(\mathrm{GA})_{26}$ & 168 & 63 & \\
\hline Fes 1394 & GCCTTGGCTGCTTGACCTTT & GATCACACGGGAGGAGAGGA & $(\mathrm{CT})_{18}$ & 181 & 60 & 0 \\
\hline Fes 1403 & CTAAGGCTCTTACATCTCAC & ACСTTCTGAGCGGAGATGTG & $(\mathrm{CT})_{25}$ & 176 & 63 & \\
\hline Fes 1407 & GTGATGAGTAGTTGCCTCTG & CTTGGCTTAGACCTCTCGTA & $(\mathrm{CT})_{13} \mathrm{CA}(\mathrm{CT})_{21}(\mathrm{CA})_{9}$ & 153 & 62 & $\bigcirc$ \\
\hline Fes1409 & GATCCGGTAGGTGAACGAGA & AATCAACGCTATTGCCTTCC & $(\mathrm{GA})_{10}$ & 166 & 56 & O \\
\hline Fes 1427 & AAGAACTGTTATCGGATGGA & ATATTGGCCTATTGGAACCT & $(\mathrm{CT})_{24}$ & 183 & 57 & \\
\hline Fes 1429 & CGTGCTTACTTGTCTTCCTTTG & GTGGTGAGAAGAAGCTCATACG & $(\mathrm{TC})_{10}$ & 197 & 63 & \\
\hline Fes 1434 & TTGCTCAACGGGAGAAACTT & GTGGTGGTGGCGGCACATAG & $(\mathrm{GA})_{29}$ & 181 & 59 & \\
\hline Fes 1445 & AGAAACTGAGCCCTTAGAAC & CTACGCCTCAGTAAAGAAGA & $(\mathrm{CT})_{19}$ & 151 & 62 & \\
\hline Fes 1455 & CAAGAGCGAAGTGGGAACAT & GGAAGGTCATTTCTAGGGTC & $(\mathrm{CT})_{36}$ & 153 & 57 & \\
\hline Fes1459 & GATCAACCACCTCAGAAAATGA & ССCTCCCTTTAGTCCGTAAAAT & $(\mathrm{GA})_{40}$ & 138 & 54 & \\
\hline Fes 1461 & CTAAATGAGCAGCCСТСТCTCT & GAGAAGATGCCAAGAGTTCAAA & $(\mathrm{CT})_{18}$ & 121 & 61 & \\
\hline Fes 1466 & ATGCCAGCTCGTATCTCTTC & GATTCGGCTAAGTAACAAAG & $(\mathrm{CT})_{25}$ & 116 & 54 & \\
\hline Fes 1472 & GGGCCACCAACAGTATTAGC & TATCAAAGGAAATCGATGAC & $(\mathrm{CT})_{32} \mathrm{TT}(\mathrm{CT})_{11}$ & 215 & 49 & \\
\hline Fes 1475 & GCAGCAACATTGACAAACTA & GCGGTGCCCTGGTGGTGGTG & $(\mathrm{AG})_{28}$ & 205 & 52 & $\bigcirc$ \\
\hline Fes 1497 & GTTGGCTGACGAAGACCGAC & AAAGAGAGCGAGAGGCACTG & $(\mathrm{CT})_{33}$ & 153 & 60 & $\bigcirc$ \\
\hline Fes 1498 & GACACTTCCTTGCCTCACAT & GATCCAACAGTTACGTTCTG & $(\mathrm{CT})_{18}$ & 147 & 61 & $\bigcirc$ \\
\hline Fes 1501 & AGGAAGTAAAATGGGGGTTACA & CGTATTTTTCTCTCCCСАТСТC & $(\mathrm{GA})_{6}$ & 63 & 52 & $\bigcirc$ \\
\hline Fes 1526 & GCTCTTGACCCTTGAACACC & TTACTCTGCAGCCCGATTCT & $(\mathrm{CT})_{8}$ & 211 & 64 & \\
\hline Fes 1533 & GAAGTAGCACCATGAGAGAA & CССТCACCTAGAGTATGACC & $(\mathrm{GA})_{8}$ & 163 & 57 & \\
\hline Fes 1550 & AGACCACTTCTCGGAAAGAAAG & ACAATTTCACACAGGAAACAGC & $(\mathrm{GA})_{28}$ & 228 & 53 & \\
\hline Fes 1571 & GGAGAAGATGTACGTAGGGA & CCTTTGACCCGTGCTTTACT & $(\mathrm{GA})_{24}$ & 167 & 59 & \\
\hline Fes 1575 & GAATTAGACGATGCCTTGGA & TAGCGGGTGGTGGTTCTTCT & $(\mathrm{GA})_{8} \mathrm{AA}(\mathrm{GA})_{7} \mathrm{CA}(\mathrm{GA})_{7}$ & 243 & 61 & \\
\hline Fes 1577 & GAGGGAGAAGATGAAGTTGT & CTGCGGATTAGGGTGTGTTG & $(\mathrm{GA})_{25}$ & 172 & 59 & \\
\hline Fes 1582 & TCTGGAGAAGCTAAACCCAC & CCGCAGTTTGTAGGGAGGGA & $(\mathrm{GA})_{24}$ & 143 & 57 & O \\
\hline Fes 1583 & CTCCAAGTCAGCCCAAGAAA & GGTCCAAGGGAGAAGCGTAG & $(\mathrm{CT})_{12}$ & 210 & 62 & $\bigcirc$ \\
\hline Fes 1585 & GCCTCCTACTAGCCCTTCCA & AGCTGACCCTTGTGTCTCCC & $(\mathrm{GA})_{7}$ & 169 & 60 & $\bigcirc$ \\
\hline Fes 1589 & TCGGAACCGCCTAAATGAAA & CTCAAGCCCTAGCGAACCAC & $(\mathrm{GA})_{21}$ & 232 & 62 & \\
\hline Fes 1592 & ACATCTAAAAGAGAGCGAGAGG & TAGCGATTAAAGGTGATTGACG & $(\mathrm{CT})_{33}$ & 235 & 61 & \\
\hline Fes 1623 & CGACCGAGATTTGGTTTGAA & CGTGGTGAATTAGGGTTGTG & $(\mathrm{CT})_{13} \mathrm{AT}(\mathrm{CT})_{36}$ & 178 & 55 & $\bigcirc$ \\
\hline Fes 1627 & AAAGTATTCGTCCCCAAATC & ATCGTCTAATCCCAGCCATC & $(\mathrm{GA})_{7}$ & 189 & 55 & \\
\hline Fes 1630 & GCGTTGTTTGCTTCATCTTT & GCTCGCTACAACATGAGGAA & $(\mathrm{CA})_{8}(\mathrm{GA})_{31}$ & 207 & 61 & \\
\hline Fes 1636 & GATTGCATGCAGATTTACAGGT & ACTGACTCGGTCGAGGTAAAA & $(\mathrm{A})_{15}$ & 106 & 61 & \\
\hline Fes 1647 & TTTATATCTTGCGTGAATGA & GTGGCGACAAGACTGTGAAG & $(\mathrm{GA})_{10} \mathrm{~A}(\mathrm{GA})_{14}$ & 142 & 55 & \\
\hline Fes 1663 & GGGAAGAGAGAAACAAGGAGAA & TTATCCAAAAAGGAGCCTATGA & $(\mathrm{GA})_{5} \mathrm{GC}(\mathrm{GA})_{11}$ & 123 & 55 & \\
\hline Fes 1667 & GGAGCCTATGAGGGTTGTTG & GGGAAGTGAAATATGTGAAT & $(\mathrm{CT})_{32}$ & 142 & 57 & \\
\hline Fes 1689 & AATTGCCGTTGTTATTCCTC & CAGTAATAATGCATAGTGCC & $(\mathrm{CT})_{24}$ & 229 & 59 & \\
\hline Fes 1693 & GCATAGAACTCTGTGGTGGTGT & CCAGCAGTGACTTGCTTCTAGT & $(\mathrm{CTG})_{4}$ & 223 & 69 & $\bigcirc$ \\
\hline Fes1694 & GCTGGAAGAAGCAAACCCTC & GCACCGCCTTCTTTGACTGT & $(\mathrm{AG})_{8} \mathrm{G}(\mathrm{GA})_{9}$ & 115 & 63 & \\
\hline Fes 1709 & CAACTAGGCTCTTACATCTC & GTTATGGCCTTCTGAGTGGA & $(\mathrm{GA})_{20} \mathrm{GG}(\mathrm{GA})_{7}$ & 186 & 59 & \\
\hline
\end{tabular}


Appendix 1. (continued)

\begin{tabular}{|c|c|c|c|c|c|c|}
\hline Locus & Forward primer $\left(5^{\prime}\right.$ to $\left.3^{\prime}\right)$ & Reverse primer ( $5^{\prime}$ to $\left.3^{\prime}\right)$ & Repeat type & $\begin{array}{l}\text { Fragment } \\
\text { size (bp) }\end{array}$ & $\begin{array}{l}\text { Annealing } \\
\text { temp. }\left({ }^{\circ} \mathrm{C}\right)\end{array}$ & $\begin{array}{l}\text { Evaluation } \\
\text { test* }\end{array}$ \\
\hline Fes1721 & TAGGGTTGAAAGAGGGAAGAAG & GAGACAACAAATCCGAGCTATG & $(\mathrm{GAA})_{9}$ & 82 & 55 & \\
\hline Fes1759 & GCGCACCACCTCCATCTTCT & TGGCGCTTACCTCATCTAAC & $(\mathrm{CT})_{31}$ & 168 & 63 & \\
\hline Fes 1776 & GGTTATAACGACATGCCTTCAA & TGTTCCATCTTCAGCTATGACC & $(\mathrm{GA})_{11}$ & 127 & 63 & \\
\hline Fes 1805 & CGCCTTGTTTGACCGTCTTG & AGAAAGTAAACCCTCATCAC & $(\mathrm{CT})_{24}$ & 125 & 59 & \\
\hline Fes 1810 & CGTCTTGCTTTTCGTTTCTTCT & AGCTAAACCCACCTCAAAGGA & $(\mathrm{CT})_{17}$ & 88 & 61 & \\
\hline Fes 1816 & ACCGGAAGTGTAGTTGATAA & GCAACTCGTAGAGTCCCACA & $(\mathrm{GA})_{18} \mathrm{AA}(\mathrm{GA})_{10}$ & 166 & 55 & O \\
\hline Fes 1822 & ATTGGAACAACTGCGCCTCA & GGCACGAACTGGGCTCTTAG & $(\mathrm{GA})_{22}$ & 156 & 57 & $\bigcirc$ \\
\hline Fes 1830 & AGGAGAGAAAGGGGAGAGAGTT & ATTGTTAGCGAATTACCGACCT & $(\mathrm{GA})_{6}$ & 216 & 64 & \\
\hline Fes 1832 & TCTTCCAAGAACACCCACAA & CCCAGGACCACCCACGACAT & $(\mathrm{GA})_{23}$ & 180 & 61 & \\
\hline Fes 1838 & GAGGAAGCACAAGGAAATGA & CCCATCGTTCCTAGCTTGAG & $(\mathrm{GA})_{7} \mathrm{AA}(\mathrm{GA})_{23}$ & 176 & 59 & \\
\hline Fes 1840 & ACGACGAAGACAAATGAGGA & ATATGGACGGCCTGGATTAT & $(\mathrm{GA})_{8}$ & 245 & 62 & $\bigcirc$ \\
\hline Fes 1852 & ATGTGCTTTGCAGGCTGACT & AATGCGAAATGTTCTAATCT & $(\mathrm{CT})_{27}$ & 194 & 55 & \\
\hline Fes 1858 & ACGTGAGAGTGAAATGAGAGGA & AAAGTGAGAGGATGGGTCAGTT & $(\mathrm{GA})_{8}$ & 96 & 63 & \\
\hline Fes 1865 & GAGGGAGATGATGTGAAGACAA & CTTAGTTGACTCCCATTTGCAC & $(\mathrm{GA})_{6}$ & 199 & 59 & \\
\hline Fes 1889 & GAATGTACCAAAGTCCTGCTCA & AGTGGCTTCCTGAGTATTCCAT & $(\mathrm{TCC})_{4}$ & 185 & 63 & \\
\hline Fes 1896 & CGAACCCTCTATTTTGTTTTACG & ACTCTCCACCACCTCCATTAGT & $(\mathrm{TGG})_{4}$ & 186 & 64 & $\bigcirc$ \\
\hline Fes2008 & CCGAGGGCTTCCGTACATAG & TACAACAAGACACCGACAAA & $(\mathrm{GTT})_{7}$ & 173 & 63 & \\
\hline Fes2017 & GAAGCATGATGGGTGTTTATTC & CACTCAGTTGCTGGTCTAACCT & $(\mathrm{TG})_{7}$ & 133 & 64 & \\
\hline Fes 2222 & AGGGCAAAAGACGAACTACACT & GCTAAGCAACAAGTCCTTGACA & $(\mathrm{GGT})_{4}$ & 207 & 55 & \\
\hline Fes2239 & AACCCCCTATTGTATTTTACGC & CTAACAACCATTCСССАAАCTC & $(\mathrm{TGG})_{4}$ & 241 & 61 & \\
\hline Fes 2279 & ССССТСТСТСАСАСААТАТСАА & CACTTTTCTGGCGTTGTTGG & $(\mathrm{CCA})_{4}$ & 123 & 59 & \\
\hline Fes 2280 & ACTTGGAGATGAAGCTCTCCTG & TTAATTCATCCATATCGGCTTG & $(\mathrm{GA})_{7}$ & 87 & 53 & \\
\hline Fes 2328 & TTACCAACCGAATCTGAATC & TTGTCTTGTGCTTTCCGACC & $(\mathrm{GA})_{8} \mathrm{GG}(\mathrm{GA})_{21}$ & 242 & 60 & $\bigcirc$ \\
\hline Fes 2420 & AGAAAACTCAAATCGGGATGAT & GCGTATCAGTCGACCTTACTCA & $(\mathrm{GA})_{6}$ & 206 & 59 & \\
\hline Fes2456 & AGGGTCCAATGAGAGAGAGAGA & ACAGAACGCAAATAACACATCC & $(\mathrm{CT})_{38}$ & 245 & 55 & \\
\hline Fes 2459 & CGGGATGAGCAACTCTGACT & TTATACGCTCCACGCTTCCT & $(\mathrm{GA})_{22}$ & 230 & 49 & $\bigcirc$ \\
\hline Fes2468 & GTAGTGGAAAGGCTCGTAAA & ATGATTGAGGATGAACGTCT & $(\mathrm{GA})_{33}$ & 183 & 55 & $\bigcirc$ \\
\hline Fes 2473 & GTGACCTCGGTTGACCATCT & TCCTTGTTGATTTCGGTTTA & $(\mathrm{CT})_{19}$ & 231 & 54 & \\
\hline Fes 2476 & TCCATTCTTCACGGCTAATC & TGCCAGGCTAACTTCTTGTA & $(\mathrm{GA})_{46}$ & 205 & 47 & \\
\hline Fes2480 & TTCGTAGTTATTGTTGGTTG & TTGCATGGTAAGAACTGTTT & $(\mathrm{CA})_{7} \mathrm{GA}(\mathrm{CA})_{9}$ & 236 & 56 & $\bigcirc$ \\
\hline Fes 2481 & AGTCCTTTGGGCTTACTTAG & TTATACCAATTTTGATATGTTC & $(\mathrm{GT})_{24}$ & 123 & 61 & \\
\hline Fes2489 & ATTTCATATTTGCATTGTCC & GATCGACCACAAGAAGTAGG & $(\mathrm{GA})_{49}$ & 180 & 49 & \\
\hline Fes 2490 & TTTGTCACCAAGTTCTTGAA & ATCCCAGCTACAACAAACAT & $(\mathrm{AT})_{8} \mathrm{AGA}(\mathrm{TG})_{14}$ & 196 & 59 & \\
\hline Fes 2507 & AGGCAAGATTTCAGTTTAGG & CTTGTGCGATTATTTGAGTC & $(\mathrm{CT})_{14}$ & 129 & 55 & $\bigcirc$ \\
\hline Fes 2509 & AGACTTTGAGATGAAGCTCT & GGTTCGCTTGCTTGATTTCT & $(\mathrm{GA})_{23}$ & 326 & 57 & \\
\hline Fes 2513 & GATCAAGCATCATACCTTTA & AGCCTAACTCAGCACACTCA & $(\mathrm{AT})_{10}(\mathrm{GA})_{16}$ & 217 & 47 & \\
\hline Fes 2530 & GAGAGAACGAGAAAGAGAAGCA & ATGAGAATGGCTGGTGATAGTG & $(\mathrm{GA})_{20}$ & 189 & 63 & \\
\hline Fes 2534 & GTAATGTCCCGAGTTCTGAG & GGGAAAGATTGAATCTTGTA & $(\mathrm{CT})_{19}$ & 138 & 50 & \\
\hline Fes 2537 & AGAGAGTGCTAGGCAAACCTTC & ATTACTTGCAGGGGAGAGAGAG & $(\mathrm{CT})_{12}$ & 138 & 62 & \\
\hline Fes 2542 & GATCCGAATGTGTTAAGTAA & ATAAATCCATTGCTTCCACC & $(\mathrm{CTT})_{30}$ & 146 & 51 & \\
\hline Fes2604 & GCGATATGCTAGTCTTGTACGG & GGTATTTAGCTCTTGCTGAGTGA & $(\mathrm{CA})_{14}$ & 99 & 63 & \\
\hline Fes2644 & GGAGTAAGACCTCCACAAAGGT & GCAAGTGATAACCССТСТСТCT & $(\mathrm{GA})_{13}$ & 186 & 50 & O \\
\hline Fes 2647 & AAGGCTTACAGTTGGTCATC & GTCCCAAATAGGCATACAAA & $(\mathrm{GA})_{35}$ & 236 & 59 & \\
\hline Fes2649 & GATCCTGATTCTTGAAGAAG & CGCTTTCTATTCATTTGTCC & $(\mathrm{GAA})_{27}$ & 234 & 57 & \\
\hline Fes2666 & CCCGAGTTCCGCAGATTTGG & TCTTTGGTCGGGCTCAATAG & $(\mathrm{GA})_{14}$ & 264 & 62 & \\
\hline Fes 2675 & TTTGGGATAGAATGTACGAG & CCGTCCTACAGCAGAATAAG & $(\mathrm{CA})_{15}(\mathrm{TA})_{8}(\mathrm{GA})_{7}$ & 156 & 59 & \\
\hline Fes2695 & ACCGAGATAGAGACCGAGAT & CAAACTAACATACCCAAAGA & $(\mathrm{CT})_{20}$ & 212 & 57 & \\
\hline Fes2696 & GTCACCCAAGCTAAGAAGAT & AACGGATACAATTTGTTCTC & $(\mathrm{GA})_{33}$ & 209 & 60 & \\
\hline Fes 2713 & AGTCTCCGGACTTACTTATT & CCTATTACCGGTTTAGTTAT & $(\mathrm{GT})_{8}$ & 112 & 57 & \\
\hline Fes 2741 & CGGTTTAATCGACATCACAT & AGATTGAGGTAAGTCCGTAG & $(\mathrm{AC})_{10}$ & 124 & 50 & $\bigcirc$ \\
\hline Fes 2742 & ATGCGGAGGAAATGTGAGAC & ТСТТССТТСААТСТТАТССС & $(\mathrm{AG})_{13} \mathrm{TGT}(\mathrm{GA})_{6}$ & 200 & 55 & \\
\hline Fes 2743 & AATGCGCTGCGGTAAACCTA & TTGGAGTATTCGCTGGTGTG & $(\mathrm{GT})_{10}$ & 263 & 55 & $\bigcirc$ \\
\hline Fes 2748 & TATGCTGCGGCTAATAAACACT & CATCCATTTAGAGAGAGAGAGAG & $(\mathrm{CT})_{20}$ & 77 & 47 & \\
\hline Fes 2751 & AAGATTTGAGGGATGAGGTGAT & CTCCCAACCGATATAAACACAA & $(\mathrm{GA})_{18}$ & 210 & 61 & $\bigcirc$ \\
\hline Fes2798 & TTCACATATATTCGGCGTAT & TGAAATAACCGGGAGTAGAT & $(\mathrm{GT})_{10}$ & 170 & 55 & $\bigcirc$ \\
\hline Fes 2802 & ACCGCGATTATGAGTTTAGA & CTAGTGGGAAGCGATTGGTA & $(\mathrm{GA})_{25}$ & 262 & 62 & $\bigcirc$ \\
\hline Fes2809 & ATCAACACAAACAATCAAGC & TAGAATTCCCGTCCTTAGGT & $(\mathrm{CA})_{13}$ & 277 & 58 & $\bigcirc$ \\
\hline Fes2819 & AGGTATGAGGCGAACGAGAC & CCGCCGAATATTTCTTCCAC & $(\mathrm{CT})_{20}$ & 280 & 54 & \\
\hline Fes 2822 & CTCCACCTTGATGTAAATGA & AGGGACATATCTCTTGGTTG & $(\mathrm{AT})_{7}(\mathrm{GT})_{7} \mathrm{GC}(\mathrm{GT})_{22}$ & 297 & 55 & \\
\hline Fes 2832 & CCAGAACCTAGAAATCGCAGTA & TGGTGAGTATTAAGGGTCGAGA & $(\mathrm{GA})_{23}$ & 179 & 63 & \\
\hline Fes2842 & AGGGCATCTACAAAATCGGTAT & TGATGTCTATGTGTGTGTGTGTG & $(\mathrm{CA})_{11}$ & 97 & 59 & \\
\hline Fes 2857 & CGTGAATTGTGGGATATGGG & TGCCACCTCAACTCACTCTC & $(\mathrm{GA})_{24}$ & 197 & 55 & $\bigcirc$ \\
\hline Fes 2864 & TGAACAACACATGATAGCCT & CGGGTCCATGTACTCATTAT & $(\mathrm{CA})_{9} \mathrm{AA}(\mathrm{CA})_{17}(\mathrm{TA})_{6}$ & 167 & 61 & \\
\hline
\end{tabular}


Appendix 1. (continued)

\begin{tabular}{|c|c|c|c|c|c|c|}
\hline Locus & Forward primer $\left(5^{\prime}\right.$ to $\left.3^{\prime}\right)$ & Reverse primer $\left(5^{\prime}\right.$ to $\left.3^{\prime}\right)$ & Repeat type & $\begin{array}{l}\text { Fragment } \\
\text { size (bp) }\end{array}$ & $\begin{array}{l}\text { Annealing } \\
\text { temp. }\left({ }^{\circ} \mathrm{C}\right)\end{array}$ & $\begin{array}{c}\text { Evaluation } \\
\text { test* }\end{array}$ \\
\hline Fes2868 & TGGAACACACATGATAGCCT & GAGTCTTCCATTCGGCATAC & $(\mathrm{CA})_{29}(\mathrm{TA})_{6}$ & 139 & 61 & \\
\hline Fes 2900 & CTAATTTGCGGGTTCCTTTAAC & GATTTGGAGGGAGAGAGAGAGA & $(\mathrm{CT})_{18}$ & 75 & 52 & 0 \\
\hline Fes2953 & CGGCAGCTTAGTTTATTGGATT & AAGTCCCATCATGCAACTACAA & $(\mathrm{AT})_{5}(\mathrm{GT})_{12}$ & 105 & 63 & \\
\hline Fes2960 & CCCAATGTGAGAGAGAGAGAGA & TCGATCAATACCTACCTACCTACC & $(\mathrm{CT})_{12}$ & 82 & 55 & \\
\hline Fes2976 & AGAAAGAATCGACGTGAGTG & CCCGTTCCAACCCTTCGTTC & $(\mathrm{GA})_{18}$ & 191 & 50 & 0 \\
\hline Fes2977 & TTCAAGGGAGGCATTGGAAC & CACGATTACTTTGCCCGACC & $(\mathrm{GA})_{39}$ & 200 & 62 & \\
\hline Fes2980 & ATTTGCATTGTCCCTAATGAGA & ATCGACCACAAGAAGTAGGTCA & $(\mathrm{GA})_{50}$ & 174 & 62 & \\
\hline Fes3075 & AGTTGAAACATGCTTTTCAGGA & TAATTTGTGAACCAGAGCGAGT & $(\mathrm{TTC})_{4}$ & 249 & 63 & 0 \\
\hline Fes3095 & GCGACTCTGATAGCGAGGGT & GGGTTCGGTTGACTAGAATG & $(\mathrm{GA})_{24}$ & 114 & 57 & \\
\hline Fes 3108 & GACCTTGACTTCTTTGACCG & GTAAGCATTCTAGGCATTTG & $(\mathrm{AT})_{7}(\mathrm{GT})_{11} \mathrm{CT}(\mathrm{GT})_{11}$ & 147 & 58 & 0 \\
\hline Fes 3123 & AGAGTTCACGGACGAGATTT & GCTCCACTTAGAGGTTCCTT & $(\mathrm{GAA})_{32}$ & 159 & 63 & \\
\hline Fes3141 & ACTTCGACGCCAACATTTCT & СCCTAAGATGTGCTAAGATT & $(\mathrm{GT})_{9}(\mathrm{GA})_{22}$ & 192 & 60 & 0 \\
\hline Fes3148 & ATGTTCTAATCTAATCTCCC & TTGCCTCCGGTTGAGTATTT & $(\mathrm{GA})_{36}$ & 168 & 57 & \\
\hline Fes3164 & CATTGGCTAAAGGTACAGGA & CAACACATGCTATTAGACGG & $(\mathrm{GA})_{17}$ & 195 & 55 & 0 \\
\hline Fes3167 & CCTCGTGTCACCAAATAAGT & TTGCTCTAACCTGCGGTAAA & $(\mathrm{GT})_{7} \mathrm{CT}(\mathrm{GT})_{7}$ & 207 & 63 & \\
\hline Fes 3177 & CTGTCCACACTGTCGCTCTT & GTCCCACCCATACAAATCAG & $(\mathrm{AC})_{6} \mathrm{G}(\mathrm{CA})_{6}$ & 181 & 63 & 0 \\
\hline Fes3214 & CGTACTCAGCAGGAGATGAC & TTGACCGCAATTTCCAGAAT & $(\mathrm{GA})_{19}$ & 208 & 59 & $\bigcirc$ \\
\hline Fes3237 & AGATGACTGAGATTAGACGA & AAAGCATTACACTTCAAACA & $(\mathrm{CA})_{49}$ & 194 & 47 & \\
\hline Fes3239 & ACTCGGATATTATTCTCGTTTGT & TGATGAAATCTTTTCAAAACACC & $\begin{array}{l}(\mathrm{AT})_{5}(\mathrm{GT})_{8} \mathrm{GC}(\mathrm{GT})_{4} \\
\mathrm{GC}(\mathrm{GT})_{4}(\mathrm{GC})_{5}\end{array}$ & 175 & 57 & \\
\hline Fes3265 & СTTTTCTTTCAACCATGTACGC & GATAGGCAATTAGAGCGTGAGTT & $(\mathrm{CT})_{16}$ & 145 & 50 & 0 \\
\hline Fes3309 & AAACACCTAACTCCGAACAACC & TATTGATGCACACACACACACA & $(\mathrm{GT})_{14}$ & 156 & 63 & \\
\hline Fes3331 & CCATGAAAGAACACTTTGAACA & TTAAATCCACACCAGGAATTTG & $(\mathrm{AG})_{14}$ & 145 & 50 & 0 \\
\hline Fes3340 & CATACACCACAACAGAACGATG & TTGGTTTTGCATTAGAGAGCAC & $(\mathrm{GA})_{18}$ & 114 & 61 & \\
\hline Fes3393 & TAAATGAAGCGACAAGAGAACG & TGTCTATCCCAAGCAATCATCT & $(\mathrm{CTT})_{7}$ & 117 & 59 & \\
\hline Fes3443 & ACCGCGATTATGAGTTTAGACA & TTTGGTGTGGTGACTCTCTCTC & $(\mathrm{GA})_{17}$ & 113 & 57 & \\
\hline Fes 3508 & GAGAAGAAGTGTTAGCGGTGTG & ACTCTTAAACGCAATGATGGTG & $(\mathrm{GT})_{12}(\mathrm{GA})_{10}$ & 133 & 62 & 0 \\
\hline Fes3531 & ACATCACCACCATAACCTCAAG & АТTCTCTCTCСТGСТCСАТСТC & $(\mathrm{CAA})_{4}$ & 183 & 63 & \\
\hline Fes3593 & TCCGTTCCATCGTTAGTTAGTG & TCAAGATATCTCCCAAGCAGAA & $(\mathrm{TC})_{7}$ & 184 & 59 & \\
\hline Fes 3663 & TTTTCCGCTTTTCACTCTATGA & AGTTTAGGTAGCGAGGCTTGAG & $(\mathrm{CT})_{13}$ & 249 & 59 & $\bigcirc$ \\
\hline Fes3664 & ACTCGGTCAAAGTGTTCTTCGT & GAGGTCTTAATCTGTTGCATCC & $(\mathrm{CT})_{14}$ & 242 & 61 & 0 \\
\hline Fes3684 & ATTATCAAGGGCAAAGGAACAC & ATTGTTGTAGTTCCGGTTGTTG & $(\mathrm{AAC})_{5}$ & 80 & 63 & \\
\hline Fes3714 & TCATATTGTGTGTTTTGATGTCTT & AACATGCAGGGAAACTACAACA & $(\mathrm{GT})_{7}$ & 117 & 63 & \\
\hline Fes3768 & AACCTCAAACCCGTCCTATAAA & TTGGACTTTTGTGTAAGCATGA & $(\mathrm{AC})_{10}$ & 210 & 60 & 0 \\
\hline Fes4011 & ACTCGTGGGCCAAGTAATAAGT & GATATATTGGCGAGTGCTTGAG & $(\mathrm{GT})_{11}$ & 117 & 61 & \\
\hline Fes4014 & ATCCAAGCTCTTCAATGCTACA & GTGCAGCTCACTTTTCACTCTT & $(\mathrm{GA})_{22}$ & 179 & 63 & \\
\hline Fes4021 & TTTTGCTCCTGTCACGAACTAT & GAATCTTACCCGATTCCAAGAC & $(\mathrm{CT})_{15}$ & 152 & 65 & \\
\hline Fes4034 & AGAGAAGTGAAAACCAACGTGA & TTCCTAGCTTGAGGTGACAAGA & $(\mathrm{GA})_{15}$ & 125 & 63 & \\
\hline Fes4036 & ATAACAAGCATCGTCATTGGAG & CCAAATTGAAACCGAACTTACA & $(\mathrm{GA})_{40} \mathrm{GGA}(\mathrm{G})_{10}$ & 184 & 60 & 0 \\
\hline Fes4037 & CACGTAAAAGTGAAATGAGAGGA & GGGGCAATGAGTAACAAGATTT & $(\mathrm{GA})_{6}$ & 180 & 50 & \\
\hline Fes4045 & GTACACAGCCGTTACAGACGTT & ACAATTTCACACAGGAAACAGC & $(\mathrm{CT})_{11}$ & 296 & 46 & 0 \\
\hline Fes4050 & AGGTGTGACCCCTTTAATGAAT & TACAACCAAGGCATACAAGCTC & $(\mathrm{GT})_{19} \mathrm{CT}(\mathrm{GT})_{7}$ & 220 & 55 & \\
\hline Fes4068 & ATATTCGACAAGCGAATGAAGA & GATATCCACAAACGGGTCAACT & $(\mathrm{GA})_{37}$ & 190 & 61 & \\
\hline Fes4109 & TCGAGTCCTAAAGTCAGACCAA & TGGACCAGTGATTGGTGACTAT & $(\mathrm{GA})_{33}$ & 224 & 63 & \\
\hline Fes4111 & CAAGGGAGAAGAGGAGAAgAAA & CTTCATCCCTTACCTCTGATGA & $(\mathrm{GA})_{15}$ & 103 & 61 & \\
\hline Fes4113 & CAGAGGAGTTGTTGCTTCCATA & ACCACCAACAATTTGCTTTTC & $(\mathrm{GA})_{21}$ & 113 & 59 & \\
\hline Fes4123 & TCGAGTCCTAAAGTCAGACCAA & TGGACCAGTGATTGGTGACTAT & $(\mathrm{GA})_{35}$ & 228 & 63 & \\
\hline Fes4125 & TGAGGGAGAAGATGAAGCTGTA & GTTTCTGAGATGAGTGCTGAGG & $(\mathrm{GA})_{21}$ & 235 & 63 & \\
\hline Fes4138 & TGAAGGAGAGGAAGTGAAATGA & TGTTGAGTGACGGGATTTTATC & $(\mathrm{GA})_{20}$ & 235 & 61 & \\
\hline Fes4150 & TGTTGGATTTCCCCACAAAG & AAGTTGTCTTGATTGCAACTGG & $(\mathrm{GGA})_{5}$ & 111 & 49 & \\
\hline Fes4151 & CACAACAGCAACTCAACAACAT & CCGACTTCCTTATGGTTTTCTT & $(\mathrm{GA})_{18}$ & 220 & 62 & \\
\hline Fes4175 & TGTGTTAACCAAGTGAGTTTTGG & CCATAGTGTATATGTGATGGTCCT & $(\mathrm{CA})_{15} \mathrm{CG}(\mathrm{CA})_{6}$ & 248 & 60 & \\
\hline Fes4617 & AGGGAAGGGGAAAGATTGAA & GGCACTAGACCACCAAACATAA & $(\mathrm{GA})_{10}$ & 188 & 51 & \\
\hline Fes4620 & GCTAAGCGTGATATTGGAGTTG & TGCCTGGAATAGAGCTAATGAA & $(\mathrm{ATG})_{4}$ & 235 & 62 & $\mathrm{O}$ \\
\hline Fes4636 & GCCTCAGAAAATGACACAAAAT & GATCTAGGAGAGGATGAACGTC & $(\mathrm{GA})_{45}$ & 187 & 45 & \\
\hline
\end{tabular}

* $\bigcirc: 54$ primer pairs used for the evaluation of their ability to detect microsatellite variation in common buckwheat populations and to be utilized in seven related Fagopyrum species 